\title{
Symmetries of microcanonical entropy surfaces
}

\author{
Hans Behringer \\ Institut für Theoretische Physik I, Universität Erlangen-Nürnberg, D - 91058 \\ Erlangen, Germany \\ Institut Laue-Langevin, F - 38042 Grenoble, France \\ E-mail: Hans.Behringer@physik. uni-erlangen.de
}

\begin{abstract}
Symmetry properties of the microcanonical entropy surface as a function of the energy and the order parameter are deduced from the invariance group of the Hamiltonian of the physical system. The consequences of these symmetries for the microcanonical order parameter in the high energy and in the low energy phases are investigated. In particular the breaking of the symmetry of the microcanonical entropy in the low energy regime is considered. The general statements are corroborated by investigations of various examples of classical spin systems.
\end{abstract}

PACS numbers: 05.50.+q, 64.60.-i, 75.10.-b

\section{Introduction}

Statistical mechanics relates the macroscopic thermodynamics of physical systems to its microscopic properties. The starting point of the statistical description is the density of states or equivalently the microcanonical entropy which is usually transformed to the canonical partition function. In recent years the statistical properties of various systems have been deduced directly from the microcanonical entropy [1, 2]. In particular first order phase transitions 3, 5, 4, and critical phenomena have been extensively analysed [6. 17. Those investigations reveal that certain properties of phase transitions can already be observed in finite systems making the use of the microcanonical approach advantageous. Note however that the choice of the appropriate ensemble is strongly related to the experimental setup of the physical system. Further focus is laid on the structure of the entropy surface as a geometrical object 8, 9, 10] and the question of the equivalence of the different statistical ensembles [11, 12, 13, 14. Note also that apart from the microcanonical ensemble precursors of phase transitions of an infinite system in the corresponding finite system can be investigated from different perspectives such as the distribution of Yang-Lee zeros of the finite system partition function [15] or the topology properties of the configuration space [16].

The topic of the present paper are the symmetries of the microcanonical entropy surface of finite spin systems. These symmetry properties are deduced from the symmetries of the microscopic Hamiltonian. Additionally they allow statements 
about the microcanonically defined order parameter. The order parameter in the microcanonical ensemble exhibits the characteristics of spontaneous symmetry breaking and its symmetry properties are related to those of the microcanonical entropy surface.

The work is organised in the following manner. The second section contains a recapitulation of the some of the basic concepts of the investigation of physical systems in the microcanonical approach. In the third section the question of the symmetries of spin systems and of the corresponding consequences on the microcanonical entropy is discussed. The general findings are investigated for particular examples of classical spin systems in the fourth section. The entire treatment is formulated in the language of magnetic spin systems although the statements are more general and apply to other physical situations as well.

\section{Spin systems in the microcanonical ensemble}

Consider a subset $\mathcal{P}$ of $N$ points in the $d$-dimensional space $\mathbb{Z}^{d}$ with a local spin degree of freedom $\sigma_{i}$ at each lattice site $i$. On the microscopic level a physical system is defined by its Hamiltonian $\mathcal{H}$. The Hamiltonian contains all contributions to the total energy of the system originating from all possible internal interactions of the spin variables $\sigma_{i}$. For an isolated system, i.e. no external field is applied to the spin variables, and pair interactions only the general Hamiltonian can be written as

$$
\mathcal{H}(\sigma)=\sum_{(i, j) \in \mathcal{P} \times \mathcal{P}} c_{i j} \mathcal{I}\left(\sigma_{i}, \sigma_{j}\right) .
$$

The pair interaction of the two spins $\sigma_{i}$ and $\sigma_{j}$ is given by $\mathcal{I}$. Prominent examples of the interaction term $\mathcal{I}$ are the Ising model with $\mathcal{I}\left(\sigma_{i}, \sigma_{j}\right)=\sigma_{i} \sigma_{j}$ and the $q$-state Potts

model with $\mathcal{I}\left(\sigma_{i}, \sigma_{j}\right)=\delta_{\sigma_{i}, \sigma_{j}}$. The Ising spins can take on the values $\sigma_{i}= \pm 1$ whereas the Potts spins can be in the states $\sigma_{i}=1, \ldots, q$. The coupling constants $c_{i j}$ describe the strength of the spin interactions of the various sites $i$ and $j$. These constants also define the range of the interaction. Note that the boundary conditions of the finite subset $\mathcal{P}$ in $\mathbb{Z}^{d}$ are also specified by the constants $c_{i j}$. To describe the magnetic properties of a classical spin system the total magnetisation as a further macroscopic quantity is used. The magnetisation of a given spin configuration $\sigma$ is related to an operator $\mathcal{M}$. In general the magnetisation operator is a multicomponent object:

$$
\mathcal{M}=\left(\mathcal{M}_{1}, \ldots, \mathcal{M}_{n}\right)
$$

with $n$ being the number of components. For most classical spin systems the magnetisation is just the sum of the local spin variables

$$
\mathcal{M}(\sigma)=\sum_{i \in \mathcal{P}} \sigma_{i}
$$

but it might also be a more complicated linear function of the spins $\sigma_{i}$. For an antiferromagnetic spin model for example the operator $\mathcal{M}$ denotes the staggered magnetisation. In general the operator $\mathcal{M}$ describes a macroscopic quantity that allows 
the definition of the microcanonical order parameter (see relation (9) below). The following sections stick to the language of magnetic systems.

The two variables energy $E$ and magnetisation $M$ specify a so-called macrostate of the magnetic system. For a microstate $\sigma$ these two quantities are obtained by applying the operators $\mathcal{H}$ and $\mathcal{M}$. Several different microstates $\sigma$ can belong to the same macrostate $(E, M)$. Let $\Gamma_{N}$ be the phase space of all possible microstates of a physical system of $N$ spins on a $d$-dimensional hypercube with linear extension $L$. The density of states of a discrete spin system is defined by

$$
\Omega\left(E, M, L^{-1}\right)=\sum_{\sigma \in \Gamma_{N}} \delta_{E, \mathcal{H}(\sigma)} \delta_{M, \mathcal{M}(\sigma)}
$$

where the Hamiltonian $\mathcal{H}$ and the magnetisation operator $\mathcal{M}$ give the internal energy and the magnetisation of the microstate $\sigma$. For a discrete spin model with discrete values for $E$ and $M$ the density of states $\Omega$ is the number of different microstates which are compatible with a specified macrostate.

The density of states is the starting point of the statistical description of thermostatic properties of a physical system. These properties are deduced from the corresponding thermodynamic potential. The set of natural variables on which this potential depends is determined by the physical context. For a magnetic system that is isolated from any environment the proper natural variables are the energy $E$ and the magnetisation $M$. The thermodynamic potential of an isolated system is the microcanonical entropy

$$
S\left(E, M, L^{-1}\right)=\ln \Omega\left(E, M, L^{-1}\right) .
$$

Here and in the following natural units with $k_{B}=1$ are used.

In the conventional approach the system is coupled to an (infinitely) large reservoir so that it can exchange energy and magnetisation with its surrounding. The corresponding thermodynamic potential, the Gibbs free energy, is connected to the canonical partition function

$$
Z\left(\beta, \beta h, L^{-1}\right)=\sum_{(E, M)} \Omega\left(E, M, L^{-1}\right) \exp (-\beta(E-h M))
$$

by the relation

$$
G\left(\beta, \beta h, L^{-1}\right)=-\frac{1}{\beta} \ln Z\left(\beta, \beta h, L^{-1}\right) .
$$

The external magnetic field is denoted by $h$ and $\beta$ is the inverse temperature. In the following however the microcanonical instead of the canonical approach to the thermostatic properties of physical systems is investigated.

The starting point of the microcanonical analysis of finite classical spin systems is the intensive microcanonical entropy

$$
s\left(e, m, L^{-1}\right):=\frac{1}{L^{d}} S\left(L^{d} e, L^{d} m, L^{-1}\right)
$$


of a system. In this quantity the trivial size dependence of the entropy is divided out, nevertheless $s$ will still show a non-trivial dependence on the system size. The intensive energy and magnetisation are defined by $e:=E / L^{d}$ and $m:=M / L^{d}$.

The spontaneous magnetisation of a finite microcanonical system with length $L$ for given energy $e$, i.e. the magnetisation in thermostatic equilibrium, is defined by

$$
m_{s p}(e): \Longleftrightarrow s\left(e, m_{s p}(e)\right)=\max _{m} s(e, m) .
$$

The spontaneous magnetisation of a finite system for given energy is the value $m$ at which the entropy becomes maximum for the fixed value of $e$, i.e. the macrostate $(e, m)$ that maximises the density of states defines the equilibrium macrostate. The non-vanishing multicomponent spontaneous magnetisation of the low energy phase defines a direction in the order parameter space:

$$
m_{s p}(e)=\left(m_{s p}^{(1)}(e), \ldots, m_{s p}^{(n)}(e)\right)=\left|m_{s p}(e)\right| \mu^{(0)}
$$

where $\mu^{(0)}$ is a unit vector. It should be noted that several equivalent maxima of the entropy may exist for a given energy $e$. This appearance of different but equivalent equilibrium macrostates is related to spontaneous symmetry breaking (see below). The spontaneous magnetisation defines the order parameter. For the high energy phase the microcanonical order parameter is zero reflecting a phase with high symmetry. The investigation of finite spin systems within the microcanonical approach reveals that the microcanonically defined order parameter (92) is indeed zero for energies above a critical value $e_{c}$ and becomes non-zero below this energy [7. Near the energy $e_{c}$ the magnetisation exhibits a square root dependence on the energy difference $e-e_{c}$ for all finite system sizes. The singular behaviour of the order parameter of a finite microcanonical system can therefore be characterised by a critical exponent $\tilde{\beta}=1 / 2$. Here some comments on the used language are necessary. The magnetisation of the finite system is made up of discrete data points. The energy dependence of this curve for small magnetisations is most suitably described by a continuous square root function. The foot of this curve defines the critical energy $e_{c}$ of the finite system.

The abrupt emerging of a finite order parameter at the critical value $e_{c}$ indicates the transition to an ordered phase with lower symmetry. Although the physical quantities like the order parameter or the susceptibility show singularities [7] at the transition point that are typical of phase transitions there is no phase transition in a finite microcanonical system. The microcanonical entropy of finite systems is expected to be an analytic function of its natural variables and hence a phase transition in the narrow sense does not take place. However, for all the author knows the analyticity of the microcanonical entropy is not yet proven, nevertheless it seems natural to assume this property regarding the analyticity of the canonical potential of finite systems. The use of the expressions phase transition and critical to describe properties of theories that account for singular behaviours in physical quantities but that are based on analytic potentials is familiar in the context of molecular field approximations. 


\section{Symmetry properties of the entropy}

A physical system specified on a microscopic level by its Hamiltonian $\mathcal{H}$ may exhibit certain symmetries. $\neq$ A symmetry transformation $g$ is defined by the fact that it leaves the interaction energy of all configurations $\sigma$ of the phase space $\Gamma_{N}$ invariant, i.e. for all microstates one has

$$
\mathcal{H}(\sigma)=\mathcal{H}(g(\sigma))
$$

One distinguishes between space symmetries and internal isospin symmetries. In the following the group $G_{L}$ of space symmetries of the lattice $\mathcal{P} \subset \mathbb{Z}^{d}$ is not further regarded. The Hamiltonian may also be invariant under transformations that act solely on the internal degrees of freedom $\sigma_{i}$. Such a transformation $g_{S}$ maps the spin variable $\sigma_{i}$ onto the new spin variable $g_{S}\left(\sigma_{i}\right)$. The map $g_{S}$ transforms the spins of different lattice sites in the same way constituting therefore a global internal symmetry. The set of these internal symmetry transformations defines the isospin group $G_{S}$ of the spin model. The total symmetry group of the Hamiltonian $\mathcal{H}$ is then given as the direct product $G=G_{L} \otimes G_{S}$. Although the Hamiltonian is invariant under the transformations of $G_{S}$, the magnetisation $\mathcal{M}(\sigma)$ need not be identical to the magnetisation $\mathcal{M}\left(g_{S}(\sigma)\right)$ of the transformed configuration $g_{S}(\sigma)$. An element $g_{S}$ of the group $G_{S}$ induces a transformation

$$
\mathcal{M}(\sigma) \stackrel{g_{S}}{\longmapsto} \mathcal{M}\left(g_{s}(\sigma)\right)
$$

on the magnetisation space spanned by the components $\mathcal{M}_{l}(\sigma)$ with $l=1, \ldots, n$. For physically relevant magnetisation operators $\mathcal{M}$ (see the discussed examples in Sec. 4) this induced transformation defines a $n$-dimensional representation $D\left(G_{S}\right)$ of the group $G_{S}$ by the correspondence

$$
g_{S} \longmapsto D\left(g_{S}\right)
$$

with $D\left(g_{S}\right)$ denoting the induced map. The transformed magnetisations $\mathcal{M}\left(g_{S}\left(\sigma_{1}\right)\right)$ and $\mathcal{M}\left(g_{S}\left(\sigma_{2}\right)\right)$ of any two different configurations $\sigma_{1}$ and $\sigma_{2}$ of the same macrostate $M$ are then identical and thus $g_{S}$ does induce unambiguously the map $D\left(g_{S}\right)$ onto the magnetisation space. Note however that for an arbitrarily chosen magnetisation operator $\mathcal{M}$ the transformed magnetisations of two different configurations $\sigma_{1}$ and $\sigma_{2}$ of a given macrostate $M$ may not be identical. In such a situation the transformation (12) does not induce a representation of the invariance group $G_{S}$ as the map $D\left(g_{S}\right)$ is not defined unambiguously for all $g_{S}$ in $G_{S}$. Nevertheless there might be a non-trivial subgroup $\tilde{G}_{S}$ for which the transformation (12) induces a representation $D\left(\tilde{G}_{S}\right)$. In the following however it is assumed that the representation comprises the whole invariance group $G_{S}$.

The symmetry property of the Hamiltonian is reflected by a symmetry of the microcanonical entropy as a function of the magnetisation components. Applying the symmetry transformation $g_{S}$ the macrostate $(e, m)$ is then mapped onto the macrostate $\ddagger$ Symmetry properties of classical spin systems are treated in the textbook [17]. 
$\left(e, D\left(g_{S}\right)(m)\right)$ and a microstate $\sigma$ which is compatible with the macrostate $(e, m)$ is mapped onto the new configuration $g_{S}(\sigma)$. This transformed configuration is compatible with the transformed macrostate $\left(e, D\left(g_{S}\right)(m)\right)$. This is obvious from the above discussion of the induced representation. Hence any microstate belonging to the original macrostate is mapped onto a configuration of the new macrostate. On the other hand consider a configuration $\sigma^{\prime}$ of the new macrostate $\left(e, D\left(g_{S}\right)(m)\right)$. The configuration $g_{S}^{-1}\left(\sigma^{\prime}\right)$ has magnetisation $D^{-1}\left(g_{S}\right)\left(D\left(g_{S}\right)(m)\right)=m$ and contributes therefore to the macrostate $(e, m)$. Thus there are as many configurations belonging to the macrostate $(e, m)$ as there are microstates compatible with the macrostate $\left(e, D\left(g_{S}\right)(m)\right)$. This results in the symmetry property

$$
s\left(e, D\left(G_{S}\right)(m)\right)=s(e, m)
$$

of the microcanonical entropy surface.

In the following it is assumed that the group $G_{S}$ is finite and that the representation $D\left(G_{S}\right)$ is irreducible. In a physical situation where the representation $D\left(G_{S}\right)$ is reducible it is always possible to decompose it into its irreducible contributions. The different irreducible magnetisation components can be considered separately as the physical behaviours associated with them is independent from each other.

Consider the equilibrium macrostates of a spin system. Above a certain energy $e_{c}$ the system is in a phase with zero spontaneous magnetisation. Below the critical energy $e_{c}$ the system is in a stable phase with a non-zero microcanonical order parameter. The high energy phase corresponds therefore to the macrostate $(e, 0)$ that is invariant under all transformations of $D\left(G_{S}\right)$. The high energy phase possesses the same symmetry group $G_{S}$ as the Hamiltonian $\mathcal{H}$, i.e. the order parameter $m_{s p}(e)=0$ of the high energy phase is left invariant under the action of $G_{S}$ on the order parameter space. The low energy phase below $e_{c}$ has a non-zero equilibrium magnetisation $m_{s p}$. Then some map $D\left(g_{S}\right)$ has to transform $m_{s p}$ onto a magnetisation $D\left(g_{S}\right)\left(m_{s p}\right)$ that is not identical to $m_{s p}$. If this was not the case the representation would not be irreducible. The low energy phase has consequently a smaller symmetry $G$ than the high energy phase. The group $G$ is a subgroup of $G_{S}$ whose representation $D(G) \subset D\left(G_{S}\right)$ leaves the non-zero spontaneous magnetisation $m_{s p}$ invariant, i.e.

$$
D(G)\left(m_{s p}\right)=m_{s p} .
$$

In view of the decomposition (10) of the $n$-dimensional spontaneous magnetisation $m_{s p}(e)$ into an energy dependent modulus $\left|m_{s p}(e)\right|$ (the actual order parameter) and a fixed direction $\mu^{(0)}$ in the order parameter space this property means that the direction $\mu^{(0)}$ is left invariant by the representation $D(G)$. The symmetry of the high energy phase $G_{S}$ is spontaneously broken down to the symmetry $G$ of the low energy phase characterised by a non-zero order parameter. $\S$

The group $G_{S}$ can be decomposed into left cosets with respect to the subgroup $G$ :

$$
G_{S}=G \cup h_{1} G \cup \ldots \cup h_{u-1} G .
$$

$\S$ Group theoretical aspects of spontaneous symmetry breaking are presented in [18, 17. 
This decomposition is unique in the sense that all $h_{i} G$ are disjoint well defined sets and the number $u$ of cosets appearing in the decomposition (16) is given by the index of the group $G$ in $G_{S}$ [19, 20]. The elements $h_{i}$ labelling the distinct cosets in (16) do not belong to $G$ and are not unambiguous as any element in $h_{i} G$ can be used as a label. The transformation $D\left(h_{i}\right)$ maps the unit vector $\mu^{(0)}$ onto the new direction

$$
\mu^{(i)}=D\left(h_{i}\right)\left(\mu^{(0)}\right) \neq \mu^{(0)} .
$$

Any other element $h_{i}^{\prime}$ in $h_{i} G$ transforms $\mu^{(0)}$ also onto the direction $\mu^{(i)}$. As all macrostates that are obtained from the state $\left|m_{s p}(e)\right| \mu^{(0)}$ through a transformation from the set $D\left(G_{S}\right)$ have the same value of the entropy, i.e.

$$
s\left(e, D\left(G_{S}\right)\left(\left|m_{s p}(e)\right| \mu^{(0)}\right)\right)=s\left(e,\left|m_{s p}(e)\right| \mu^{(0)}\right),
$$

all distinct macrostates $\left\{\left|m_{s p}(e)\right| \mu^{(i)} \mid i=0, \ldots, u-1\right\}$ are equivalent stable phases below the critical energy $e_{c}$. Hence there are $u$ physically equivalent phases below the transition energy. The symmetry group $G^{(i)}$ of the phase $\left|m_{s p}(e)\right| \mu^{(i)}$ is obtained from the invariance group $G$ by a conjugation with the element $h_{i}$ :

$$
G^{(i)}=h_{i} G h_{i}^{-1} \text {. }
$$

To conclude this section the order parameter symmetry of the Ising model is shortly considered. The Hamiltonian

$$
\mathcal{H}=-\sum_{\langle i, j\rangle} \sigma_{i} \sigma_{j}
$$

of the nearest neighbour Ising model (the summation over neighbour pairs is indicated by $\langle i, j\rangle$ ) with possible spin states $\sigma_{i}= \pm 1$ is invariant under the Abelian group $G_{S}=C_{2}=\{+1,-1\}$. The entropy as a function of the one-dimensional order parameter $m$ is consequently an even function

$$
s(e,-m)=s(e, m) \text {. }
$$

The equilibrium order parameter in the low energy phase is non-zero and hence the low energy phase is only invariant under the trivial subgroup $G=\{+1\}$. The two possible order parameter branches are related to each other by the group transformation -1 . They are shown in Fig. 1 for a three-dimensional Ising system with 216 spins.

\section{Entropy surface of $q$-state models}

In this section the symmetry properties of the microcanonical entropy surfaces of the three-state Potts model and the four-state vector Potts model are investigated. Both models are examples of systems with a two-dimensional order parameter. The entropy is consequently a function of the internal interaction energy $e$ and the two order parameter components $m_{1}$ and $m_{2}$. For simplicity both models are defined on a two-dimensional square lattice with linear extension $L$ and $N=L^{2}$ lattice sites. The boundary condition is chosen to be periodic. The entropy surface is obtained by the highly efficient transition observable method 21] that allows the determination of very accurate estimates of the entropy surface. 


\subsection{Three-state Potts model}

The Potts model [22] is a possible generalisation of the Ising model. The Hamiltonian is given by

$$
\mathcal{H}=-\sum_{\langle i, j\rangle} \delta_{\sigma_{i}, \sigma_{j}},
$$

where the Potts spins $\sigma_{i}$ can take on the values $1, \ldots, q$. The case $q=2$ is equivalent to the Ising model. In two dimensions the model undergoes a second order phase transition for $q<5$.

The three-state Potts model where the spin variables $\sigma_{i}$ can take on the values 1,2 , and 3 , has a three-fold degenerate completely ordered ground state with internal energy $E=-2 N$. In one of the three ground states all $N$ spin variables are in the same state. The energy $E$ of the configurations $\sigma$ can take on any integer value in the interval $[-2 N, 0]$. The magnetisation of the system for a fixed energy is related to the numbers $N^{\left(\sigma_{i}\right)}$ of spins being in the spin state $\sigma_{i}$. As the total number of spins is fixed to be $N$ these numbers are subjected to the subsidiary condition $N^{(1)}+N^{(2)}+N^{(3)}=N$ which defines a plane in the three-dimensional space spanned by the $N^{\left(\sigma_{i}\right)}$. The possible macrostates are therefore characterised by the internal energy $E$ and the two numbers $N^{(1)}$ and $N^{(2)}$ of spins in the state 1 and 2 , respectively. In this two-dimensional plane the coordinates are chosen in such a way that the completely disordered macrostate $(N / 3, N / 3)$ where all spin states are equally likely corresponds to the magnetisation $\left(M_{1}=0, M_{2}=0\right)$. The (intensive) magnetisation $\left(m_{1}, m_{2}\right)$ with $m_{l}=M_{l} / N$ is related to the occupation numbers by the map

$$
\begin{aligned}
& m_{1}=1-\frac{3}{N} \frac{N^{(1)}+N^{(2)}}{2} \\
& m_{2}=\frac{\sqrt{3}}{2 N}\left(N^{(2)}-N^{(1)}\right) .
\end{aligned}
$$

The magnetisation of the Potts model with three spin states lies within an equilateral triangle in the $m_{1} m_{2}$ plane with the vertices at the points $(1,0),(-1 / 2, \sqrt{3} / 2)$, and $(-1 / 2,-\sqrt{3} / 2)$. These points correspond to the magnetisations of the three equivalent ground states of the model. The directions defined by the magnetisation vectors of these ground states (compare (10) ) define the symmetry lines of the equilateral triangle. This triangle is depicted in Fig. 2 ,

The Hamiltonian (22) is invariant under the permutation group $S_{3}$ which is isomorphic to the group $C_{3 v}$. The invariance group of the Potts model induces a set of linear mappings acting on the two magnetisation components $m_{1}$ and $m_{2}$. This twodimensional representation of the group $C_{3 v}$ is the irreducible representation commonly denoted by $\Gamma_{3}$. Note that this representation is also faithful. The entropy surface has therefore the symmetry property (see Fig. 2)

$$
s\left(e, \Gamma_{3}\left(C_{3 v}\right)\left(m_{1}, m_{2}\right)\right)=s\left(e, m_{1}, m_{2}\right) .
$$

The appearance of the ground state magnetisation at the vertices of an equilateral

triangle suggests that the extrema of the entropy as a function of the two magnetisation 
components for a fixed energy appear either at zero magnetisation or for non-zero magnetisations along the symmetry directions of the triangle specified by the angles $0,2 \pi / 3$, and $4 \pi / 3$. Here the angles are defined with respect to the $m_{1}$ axis on which the vertex $(1,0)$ of the equilateral triangle lies.

The entropy surface of the Potts model in two dimensions with finite linear extension $L$ for a fixed energy above the critical point $e_{c}$ at which the transition to a non-zero spontaneous magnetisation takes place exhibits a single maximum at zero magnetisation. Below $e_{c}$ three equivalent maxima show up for non-zero magnetisations. They appear along the bisectors of the equilateral triangle within which lie all possible macrostates of the Potts model for fixed energy. The extremum at zero magnetisation corresponds to a minimum of the entropy for energies below the critical point. This behaviour can be illustrated with a Potts system of linear extension $L=12$. The critical energy of the finite system with 144 Potts spins is $-1.206 \pm 0.003$. Fig. 3 shows the level curves of the density of states of the Potts model for both an energy above and below the critical value $e_{c}$. The density of states as a two-dimensional manifold for the energy below $e_{c}$ is shown in Fig. [. Note that the maxima and the minima of the density of states appear at the same magnetisations as the extrema of the entropy as the logarithm is a monotonic function.

Consider the maximum that shows up along the $m_{1}$ direction in the order parameter space. The corresponding stable low energy phase has the invariance group $G=$ $\left\{E, \sigma^{(1)}\right\} \subset C_{3 v}$. Here $\sigma^{(1)}$ denotes the reflection about the $m_{1}$ direction, similarly $\sigma^{(2)}$ and $\sigma^{(3)}$ denote the reflection about the symmetry lines defined by the angles $2 \pi / 3$ and $4 \pi / 3$. The group $C_{3 v}$ can be decomposed into left cosets with respect to $G$ giving the additional cosets $C_{3} G=\left\{C_{3}, \sigma^{(3)}\right\}$ and $C_{3}^{2} G=\left\{C_{3}^{2}, \sigma^{(2)}\right\}$. The transformation $C_{3}$ applied onto the $m_{1}$ direction gives the direction $2 \pi / 3$. The corresponding phase has the symmetry group $G^{(2)}=C_{3} G C_{3}^{-1}=\left\{E, \sigma^{(2)}\right\}$ (see relations (17) and (19)).

The three-state Potts model can be investigated analytically within the plaquette approximation 22. This approach also reveals the three-fold $C_{3 v}$ symmetry of the entropy surface for fixed energies.

\subsection{Four-state vector Potts model}

An other possible generalisation of the Ising model is the $x y$ model [23] specified by the Hamiltonian

$$
\mathcal{H}=-\sum_{\langle i, j\rangle} \vec{\sigma}_{i} \cdot \vec{\sigma}_{j}=-\sum_{\langle i, j\rangle} \cos \left(\varphi_{i}-\varphi_{j}\right)
$$

with the two-dimensional unit vector $\vec{\sigma}_{i} \in S^{1}$. The angle $\varphi_{i}$ characterises the direction of the vector $\vec{\sigma}_{i}$ in the plane. The magnetisation operator is given by

$$
\overrightarrow{\mathcal{M}}=\sum_{i} \vec{\sigma}_{i}
$$

The possible intensive magnetisations of the model for a fixed energy lie within the unit circle of the $x y$ plane. The invariance group $O(2)$ of the Hamiltonian induces the 
symmetry property

$$
s(e, O(2)(\vec{m}))=s(e, \vec{m})
$$

of the entropy which has the consequence that the entropy depends only on the modulus $|\vec{m}|$ of the magnetisation vector $\vec{m}$.

The continuous $x y$ model can be discretised by restricting the angle $\varphi_{i}$ to the values $2 \pi k_{i} / q$ with $k_{i}=0, \ldots, q-1$. The resulting model is often called $q$-state vector Potts model or $q$-state clock model. The Hamilton function of the four-state vector Potts model is

$$
\mathcal{H}=-\sum_{\langle i, j\rangle} \cos \left(\frac{\pi}{2}\left(k_{i}-k_{j}\right)\right),
$$

where the spins $k_{i}$ can take on the values $0,1,2$, and 3 and may be visualised by unit vectors with angles $0, \pi / 2, \pi$, and $3 \pi / 2$ in a two-dimensional plane. The system has four equivalent ferromagnetically ordered ground states. The possible extensive energies $E$ of the spin configurations $k$ of the system are even numbers in the interval $[-2 N, 2 N]$. The magnetisation $\left(M_{1}, M_{2}\right)$ is given by

$$
\begin{aligned}
& M_{1}=\sum_{i} \cos \left(\frac{\pi}{2} k_{i}\right) \\
& M_{2}=\sum_{i} \sin \left(\frac{\pi}{2} k_{i}\right) .
\end{aligned}
$$

With the total numbers $N^{\left(k_{i}\right)}$ of spins in the spin state $k_{i}$ of a given configuration $k$ the intensive magnetisation can be expressed as

$$
\begin{aligned}
& m_{1}=\frac{1}{N}\left(N^{(0)}-N^{(2)}\right) \\
& m_{2}=\frac{1}{N}\left(N^{(1)}-N^{(3)}\right) .
\end{aligned}
$$

The four equivalent ground states have the specific magnetisations $(1,0),(0,1),(-1,0)$, and $(0,-1)$. These points define a square in the magnetisation plane containing all possible macrostates of the four-state vector Potts model for a fixed energy.

The Hamiltonian (29) is invariant under those transformations of the group $O(2)$ that leave a square in the magnetisation space invariant. The corresponding group is the group $C_{4 v}$ with the rotations $C_{4}, C_{4}^{2}$, and $C_{4}^{3}$ about the angles $\pi / 2, \pi$, and $3 \pi / 2$. It also contains the reflections $\sigma^{(1)}$ and $\sigma^{(2)}$ about the $m_{2}$ and $m_{1}$ direction and the reflections $\sigma^{(u)}$ about the direction $m_{1}=m_{2}$ and $\sigma^{(v)}$ about the direction $m_{1}=-m_{2}$. The representation of the group $C_{4 v}$ that shows up as the symmetry operations on the magnetisation of the physical system is the two-dimensional, irreducible representation $\Gamma_{5}$. Apart from $\Gamma_{5}$ the group $C_{4 v}$ has four additional one-dimensional irreducible representations. The appearance of the spontaneous magnetisation of the ground states on the lines $m_{1}=0$ and $m_{2}=0$ suggests that the non-zero spontaneous magnetisations of the system with higher energies are along the same directions.

The four-state vector Potts model with 64 spins in two dimensions undergoes a second order phase transition at the energy $e_{c}=-0.7 \pm 0.005$. In Fig. 5 the contour 
plots for entropies above and below the critical energy are shown. The system develops four equivalent maxima along the lines $m_{i}=0$ if the energy is below the critical value. Only one maximum at zero magnetisation is present for energies above $e_{c}$. Saddle points of the entropy surface turn up along the second type of symmetry lines $m_{1}= \pm m_{2}$ for energies below $e_{c}$. The high energy symmetry $C_{4 v}$ of the four-state vector Potts model is broken down to the low energy symmetry group comprising the identity and the reflection about the direction $\mu^{(0)}$ defined by the equilibrium magnetisation through the decomposition (10).

In this subsection only the four-state vector Potts model as an example of a system with four degrees of freedom is investigated. A comparison to a different system with four degrees of freedom such as the ordinary Potts model seems to be desirable. In particular the behaviour in the low energy regime is of special interest but is left to future studies.

\section{Conclusion}

In this paper it has been shown that the isospin symmetry $G_{S}$ of the Hamiltonian of a spin system determines the symmetry properties of the microcanonical entropy surface of a finite system at constant energies. The invariance group $G_{S}$ acts thereby through the irreducible representation $D\left(G_{S}\right)$ on the magnetisation components $m_{l}$. At the magnetisations $\left\{D\left(g_{S}\right)(m) \mid g_{S} \in G_{S}\right\}$ the entropy have the same value. The microcanonical order parameter below the critical point is a vector in the order parameter space with a finite modulus and defines therefore a direction in the order parameter space for the low energy phase. The invariance group $D(G)$ of this direction is a proper subgroup of $D\left(G_{S}\right)$ reflecting the spontaneous breakdown of the symmetry of the equilibrium macrostate of the system in the low energy phase. This has been demonstrated for various spin models. The symmetry group of a spin model on the microscopical level leads already to a qualitative grasp of the microcanonical entropy surface of finite spin systems. This qualitative picture of the microcanonical entropy surface allows for example to scrutinise the consistency of estimates of the entropy that are obtained by numerical simulations. Furthermore the knowledge of exact symmetries of the entropy surface may be used to symmetrise the numerical results and thereby improve the statistics of the simulation. The fact that the various maxima of the entropy surface are physically identical can be used to restrict the investigation of the behaviour of the entropy function in the vicinity of the equilibrium macrostate to the analysis of one of the equivalent maxima.

\section{Acknowledgments}

The author would like to thank Alfred Hüller and Michel Pleimling for stimulating discussions and Efim Kats for helpful comments on the manuscript. 
Symmetries of microcanonical entropy surfaces

\section{References}

[1] A. Hüller, First Order Phase Transitions in the Canonical and the Microcanonical Ensemble, Z. Phys. B 93, 401 (1994)

[2] D.H.E. Gross, Microcanonical Thermodynamics: Phase Transitions in "Small" Systems, Singapore, 2001

[3] M. Schmidt, MC-Simulation of the 3D, $q=3$ Potts Model, Z. Phys. B 95, 327 (1994)

[4] D. H.E. Gross, A. Ecker, and X.Z. Zhang, Microcanonical Thermodynamics of First Order Transitions Studied in the Potts Model, Ann. Phys. 5, 446 (1996)

[5] D. J. Wales and R. S. Berry, Coexistence in Finite Systems, Phys. Rev. Lett. 73, 2875 (1994)

[6] M. Promberger and A. Hüller, Microcanonical Analysis of a Finite Three-Dimensional Ising System, Z. Phys. B 97, 341 (1995)

[7] M. Kastner, M. Promberger, and A. Hüller, Microcanonical Finite-Size Scaling, J. Stat. Phys. 99, $1251(2000)$

[8] D. H. E. Gross and E. Votyakov, Phase Transitions in "Small" Systems, Eur. Phys. J. B 15, 115 (2000)

[9] M. Pleimling and A. Hüller, Crossing the Coexistence Line at Constant Magnetization, J. Stat. Phys. 104, 971 (2001)

[10] M. Kastner, Existence and Order of the Phase Transition of the Ising Model with Fixed Magnetization, J. Stat. Phys 107, 133 (2002)

[11] J. T. Lewis, C.-E. Pfister, and W. G. Sullivan, The Equivalence of Ensembles for Lattice Systems: Some Examples and a Counterexample, J. Stat. Phys. 77, 397 (1994)

[12] T. Dauxois, P. Holdsworth, and S. Ruffo, Violation of Ensemble Equivalence in the Antiferromagnetic Mean-Field XY Model, Eur. Phys. J. B 16, 659 (2000)

[13] J. Barré, D. Mukamel, and S. Ruffo, Inequivalence of Ensembles in a System with Long-Range Interactions, Phys. Rev. Lett. 87, 030601 (2001).

[14] I. Ispolatov and E.G.D. Cohen, On First-Order Phase Transitions in Microcanonical and Canonical Non-Extensive Systems, Physica A 295, 475 (2001)

[15] P. Borrmann, O. Mülken, and J. Harting, Classification of Phase Transitions in Small Systems, Phys. Rev. Lett. 84, 3511 (2000)

[16] L. Caiani, L. Casetti, C. Clementi, and M. Pettini, Geometry of Dynamics, Lyapunov Exponents, and Phase Transitions, Phys. Rev. Lett. 79, 4361 (1997)

[17] G. Morandi, F. Napoli, and E. Ercolessi, Statistical Mechanics, An Intermediate Course, Singapore, 2001

[18] L. Michel, Symmetry Defects and Broken Symmetry. Configurations Hidden Symmetry, Rev. Mod. Phys. 52, 617 (1980)

[19] G. J. Lyubarski, The Application of Group Theory in Physics, London, 1960

[20] L. M. Falicov, Group Theory and Its Physical Applications, Chicago, 1966

[21] A. Hüller and M. Pleimling, Microcanonical Determination of the Order Parameter Critical Exponent, Int. J. Mod. Phys. C 13, 947 (2002)

[22] F. Y. Wu, The Potts Model, Rev. Mod. Phys. 54, 235 (1982).

[23] J. B. Kogut, An Introduction to Lattice Gauge Theory and Spin Systems, Rev. Mod. Phys. 51, 659 (1979) 


\section{Figure captions}

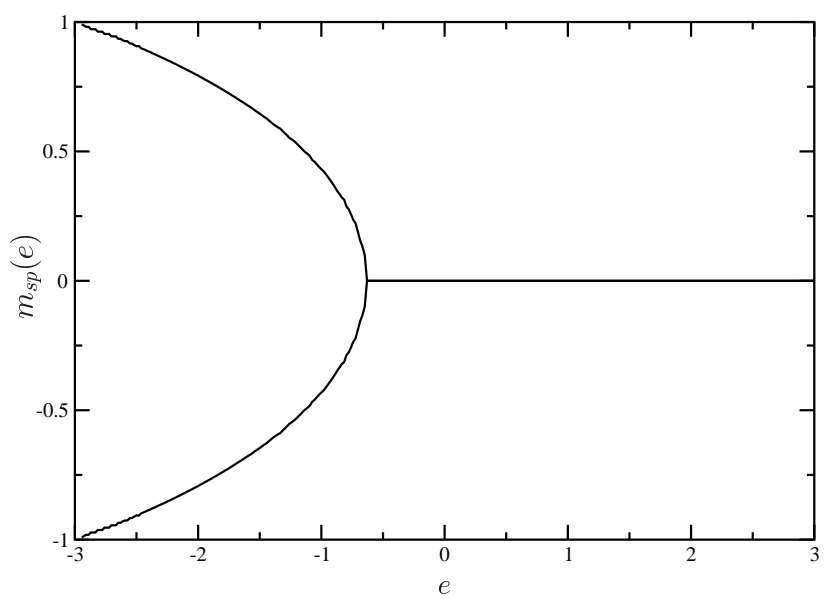

Figure 1. The two branches of the microcanonical order parameter of the three-dimensional Ising model with 216 spins as a function of the energy. The data that are obtained by a Monte Carlo simulation suggest a critical energy $e_{c}=-0.665 \pm 0.002$.

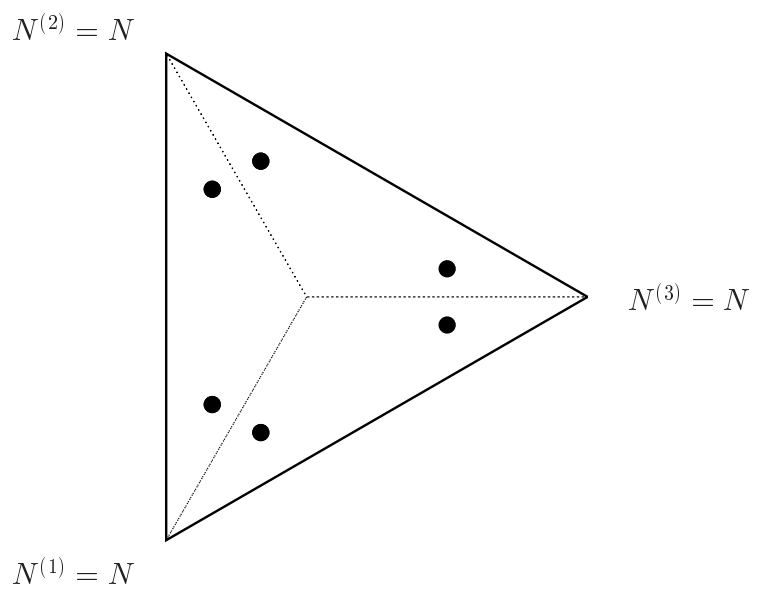

Figure 2. The equilateral triangle spanned by the magnetisations of the three different ground states of the three-state Potts model. The circles indicate macrostates with the same value of the entropy (compare relation (25)). 

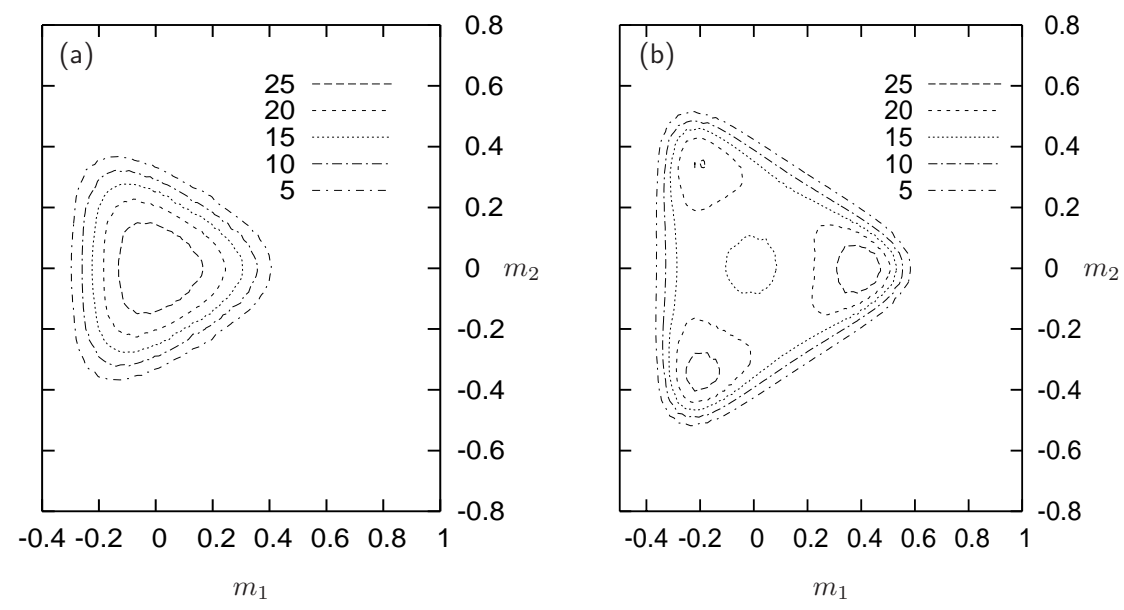

Figure 3. Level curves of the density of states of the Potts model with $L=12$ for the energies -1.166 (a) and -1.264 (b). Three equivalent maxima appear along the symmetry lines of the equilateral triangle defined by the ground state magnetisations if the energy is below the critical value -1.206 . Note that the density of states exhibits small asymmetries inevitable in Monte Carlo calculations.

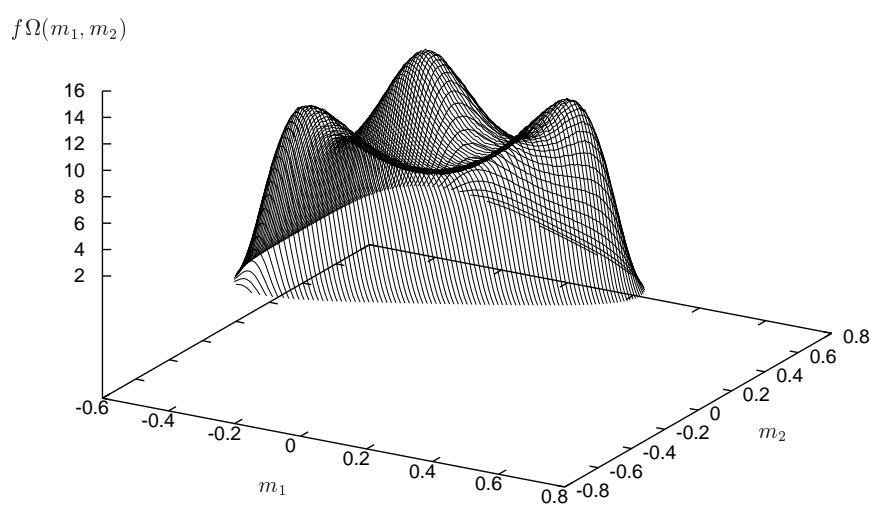

Figure 4. The density of states of the Potts model with $L=12$ for the energy $e=-1.264$ below the critical point. The density of states is shown as the maxima are more pronounced in the density of state than in the microcanonical entropy. The surface shows three pronounced maxima and reveals the threefold symmetry of the entropy surface (compare also Fig. 3.25 in [2]). The simulation gives the entropy up to a trivial additive constant. This has the consequence that the depicted density of states is only determined up to a multiplicative factor $f$. 

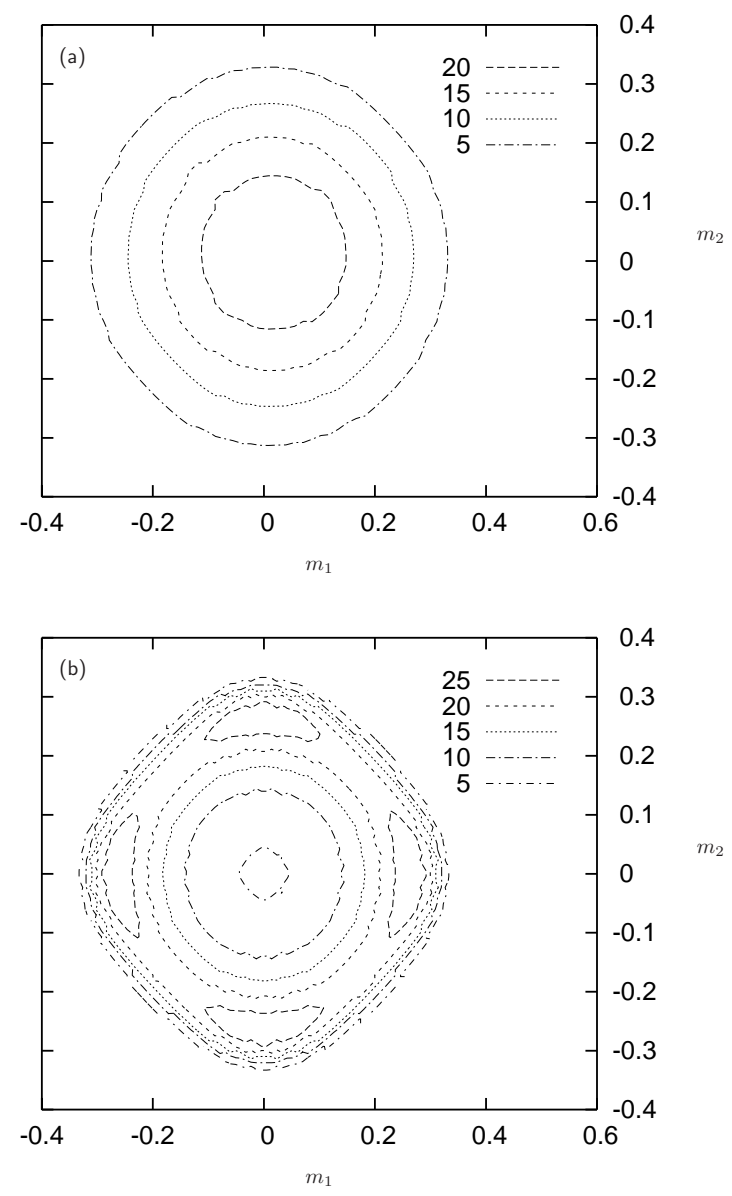

Figure 5. Level curves of the density of states of the four-states vector Potts model in two dimensions with linear extension $L=8$ for the energies -0.5 (a) and -0.968 (b). The entropy has only one maximum at zero magnetisation for energies above the critical value -0.7. A minimum at zero magnetisation and four equivalent maxima appear along the bisectors of the square defined by the ground state magnetisations if the energy is below the critical value -0.7 . 